\title{
Recidiva local tras la cirugía del cáncer de pulmón no microcrítico. Factores pronósticos
}

\author{
R. BERNAL BERNAL, A. LEÓN JIMÉNEZ, J. JAÉN OLASOLOํㄹ. E. BENÍTEZ \\ RODRÍGUEZ ${ }^{2}$, F. MATEO VALLEJO ${ }^{3}$
}

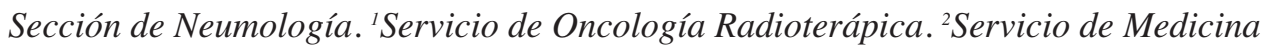

Preventiva. Hospital Universitario Puerta del Mar. Cádiz. ${ }^{3}$ Servicio de Cirugía. Hospital del Servicio Andaluz de Salud de Jerez. Cádiz

\section{RESUMEN}

Objetivos: Analizar retrospectivamente la influencia de características clínicas e histo-patológicas en la recidiva local tras cirugía del carcinoma no microcítico de pulmón.

Método: Se incluyeron en el estudio 146 pacientes seguidos durante un mínimo de 2 años. La función de supervivencia libre de enfermedad (SLE) se construyó según el método de Kaplan-Meier. La comparación univariante de la SLE se realizó mediante test de los rangos logarítmicos de Mantel-Haenszel y para el análisis multivariante se aplicó el modelo de regresión de Cox.

Resultados: La mediana de la SLE fue de 20 meses En el análisis univariante, se encontraron los siguientes factores predictores de una menor SLE: contacto radiológico del tumor con la pleura $(p=0,005)$, grado de indiferenciación histológica $(\mathrm{p}=0,03)$, afectación del margen peritumoral $(\mathrm{p}=0,001)$, afectación pleural $(\mathrm{p}=0,006)$, estadificación $\mathrm{T}$ $(\mathrm{p}=0,04)$ y estadificación $\mathrm{N}(\mathrm{p}=0,002)$. Otros factores como la edad, presencia inicial de síntomas, segundas neoplasias, presencia radiológica de espiculaciones o cavitación, punción transtorácica, tipo de cirugía, afectación del margen peribronquial, tamaño tumoral y tipo histológico, no mostraron diferencias estadísticamente significativas en la SLE. En el estudio multivariante mantienen su valor pronóstico la afectación pleural $(\mathrm{p}=0,0014)$, afectación ganglionar $(\mathrm{p}=0,02)$ y grado de diferenciación $(\mathrm{p}=0,03)$.

Conclusiones: El factor pronóstico más importante de recidiva local tras cirugía en estos pacientes fue la infiltración de la pleura, seguido de la afectación ganglionar hilio-mediastínica y el grado de diferenciación histológica.

PALABRAS CLAVE: Carcinoma no microcítico de pulmón. Cirugía. Recidiva local.
LOCAL RECURRENCE AFTER NON-SMALL CELL LUNG CANCER SURGERY. PROGNOSIS VARIABLES

\section{ABSTRACT}

Objetives: Retrospectively to analyse the influence of clinical and histo-pathological characteristics in the local recurrence after non-small cell lung cancer surgery.

Method: 146 patients were included and studied during a minimum of two years. The free survival of disease function (FSD) was constructed according to the Kaplan-Meier method. The univariante comparison of the FSD by Mantel-Haenszel means of logarithmic ranks test and the multivariante analysis by Cox regression model.

Results: In the univariante analysis 20 months of FSD and the following predicting factors of a smaller FSD: Radiological contact of the tumour with the pleura $(p=0.005)$, peritumoral margin affectation $(p=$ $0.001)$, pleural affectation $(p=0.006)$, T stratification factor $(p=0.04)$ and $N$ factor $(p=0.002)$. Other factors like age, initial symptoms, second neoplasias, radiological spiculaciones or cavitations, transtoracic puncion, type of surgery, peribronquial affectation, so large margin tumorlike and histological type, did not show statistically significant differences in the FSD. In the multivariante study they maintain its value prognosis the pleural affectation $(p=0.0014)$, ganglionary affectation $(p=0.02)$ and degree of differentiation $(p=0.03)$.

Conclusions: The most important prognosis factor of local recurrence after surgery in these patients were the infiltration of the pleura, followed of the hilio-mediastínica ganglionary affectation and the degree of histological differentiation.

KEY WORDS: Non-small cell lung carcinoma. Lung surgery. Local recurrence.

Bernal Bernal R, León Jiménez A, Jaén Olasolo J, Benítez, Rodríguez E, Mateo Vallejo F. Recidiva local tras la cirugía del cáncer de pulmón no microcrítico. Factores pronósticos. An Med Interna (Madrid) 2008; 25: 55-60.

\section{INTRODUCCIÓN}

El carcinoma broncopulmonar es el tumor diagnosticado con más frecuencia en el mundo y a la vez el que ocasiona el mayor número de muertes por cáncer. Nos encontramos ante una enfermedad de gran repercusión social, que supone además, un enorme reto para los profesionales de la salud, ya que no se ha conseguido mejorar sustancialmente su pronóstico en los últimos 20 años (1). La cirugía representa el tratamiento más apropiado para el cáncer de pulmón de células no pequeñas 
en estadio precoz. A pesar de que en grupos seleccionados de pacientes pueden obtenerse tasas de supervivencia a los 5 años superiores al $60 \%$, en conjunto más del $50 \%$ de los pacientes sometidos a resección fallecerán por una recidiva (2-4).

Aunque los sistemas de clasificación clínico-patológicos del tipo del TNM, de uso generalizado, son los que mejor se ajustan al pronóstico del paciente, existen otros factores decisivos a la hora de indicar un tratamiento $(5,6)$. Este trabajo se ha planteado para analizar otras variables no incluidas en la clasificación TNM actual que pudieran estar relacionadas con el desarrollo de la recidiva local.

\section{MATERIAL Y MÉTODO}

\section{PACIENTES Y VARIABLES}

Se realizó un estudio retrospectivo sobre 175 pacientes diagnosticados de cáncer de pulmón no microcítico, sometidos a intervención con intención curativa en el Hospital Universitario Puerta del Mar de Cádiz. Dichos pacientes fueron diagnosticados entre enero de 1995 y diciembre de 2000. Diez de estos pacientes fueron excluidos para el estudio por no cumplir un seguimiento mínimo de 2 años y 19 por fallecimiento relacionado con complicaciones posquirúrgicas o por causa de origen no neoplásico, quedando 146 pacientes válidos para el estudio.

Como posibles factores pronósticos, se analizaron las siguientes variables clínicas: edad, diagnóstico previo de otra enfermedad neoplásica en remisión completa, presencia o no de síntomas, realización de punción transtorácica y tipo de cirugía. Las características radiológicas (por TAC) analizadas fueron: presencia de espiculaciones, cavitación intratumoral y contacto tumoral con la pleura. Además, se estudiaron los siguientes factores histopatológicos: tamaño tumoral, tipo histológico, grado de diferenciación, afectación de los márgenes peribronquial y peritumoral, infiltración pleural y estadificación. Dicha estadificación se realizó de acuerdo con el Sistema Internacional de Clasificación Revisado para el Cáncer de Pulmón (7) adoptado en 1997 por el "American Joint Comittee on Cancer" (AJCC) (8) y la "Union International Contre le Cancer" (UICC) y en 1998 por la SEPAR (9).

\section{ANÁLISIS ESTADÍSTICO}

En primer lugar se realizó un estudio descriptivo de todas las variables objeto de estudio, así como pruebas de normalidad (Kolmogorov-Smirnov) de las variables cuantitativas.

La supervivencia libre de enfermedad (SLE) se calculó desde la fecha de la intervención quirúrgica hasta la primera fecha en que se constata una recidiva, bien por estudios de imagen o histopatológicos (8). Las curvas de supervivencia se construyeron de acuerdo al método de Kaplan-Meier (11) y las diferencias entre curvas de supervivencia se compararon mediante la prueba de los rangos logarítmicos (log-rank) de Mantel-Haenszel (12). El análisis multivariante de factores pronósticos respecto a la supervivencia libre de enfermedad se realizó mediante el modelo de regresión de riesgos proporcionales de Cox (13). Todos los p-valores se calcularon de forma bilateral, considerándose estadísticamente significativos valores inferiores a 0,05 .

\section{RESULTADOS}

\section{CARACTERÍSTICAS CLÍNICAS GENERALES}

El $95 \%$ de los enfermos fueron varones con una edad media de 61 años (desviación estándar 9 años). El 55\% de los pacientes eran fumadores activos de un promedio de 68 paquetes/año, el 39\% exfumadores de 70 paquetes/año y sólo el $6 \%$ eran no fumadores.

El $43 \%$ de los pacientes estaban diagnosticados de enfermedad pulmonar obstructiva crónica y un $16 \%$ habían padecido previamente otra neoplasia maligna. De éstos, la mayoría se refieren a la esfera ORL (59\%) y urológica (22\%), que comparten el tabaquismo como factor etiológico más relevante.

Desde el comienzo de los síntomas atribuibles a la enfermedad neoplásica hasta la primera sospecha clínica (radiológica) transcurre un intervalo de tiempo corto (mediana de 18 días). Desde este momento, hasta la confirmación histológica de cáncer, transcurre un intervalo similar (mediana de 20 días), y hasta la intervención quirúrgica el tiempo es algo más prolongado (mediana de 62 días). La mediana de la demora global (desde el primer síntoma hasta la cirugía) fue de 102 días.

El primer diagnóstico clínico-radiológico de sospecha de cáncer pulmonar, lo realizó el Servicio de Urgencias Hospitalario en el 26\% de los casos, el Médico de Atención Primaria en el $35 \%$ y el Especialista en el 39\%

En cuanto a los síntomas de presentación, los más frecuentes fueron la tos, hemoptisis y dolor torácico, como queda reflejado en la tabla I. Un tercio (33\%) de los pacientes no aquejaban síntomas debidos a la enfermedad y se llegó al diagnóstico por estudios radiológicos preoperatorios o relacionados con otras patologías.

\section{TABLA I}

SÍNTOMAS DE PRESENTACIÓN

\begin{tabular}{lc}
\hline Síntoma & Porcentaje \\
\hline Tos & $21 \%$ \\
Hemoptisis & $20 \%$ \\
Dolor torácico & $20 \%$ \\
Infección respiratoria & $10 \%$ \\
Pérdida ponderal & $8 \%$ \\
Fiebre & $7 \%$ \\
Disnea & $3 \%$ \\
Dolor óseo & $3 \%$ \\
Dolor de hombro & $2 \%$ \\
Otros & $6 \%$ \\
\hline
\end{tabular}

La presentación radiológica más frecuente fue la de una imagen nodular $(82 \%)$ de un tamaño medio de $46 \mathrm{~mm}$, con presencia de cavitación en el $16 \%$. Un $8 \%$ mostraban atelectasia y el $10 \%$ presentaban nódulo y atelectasia. La localización anatómica más frecuente fue el lóbulo superior (55\%) seguida del inferior (39\%) y después el medio (6\%), con discreto predominio del pulmón derecho $(56 \%)$ frente al izquierdo.

Para el diagnóstico histológico al $94 \%$ de los pacientes se les practicó una fibrobroncoscopia y al 52\% una punción transtorácica con aguja fina. La rentabilidad diagnóstica de la biopsia bronquial fue del $55 \%$ (49 positiva $+6 \%$ sospechosa) 
y de la punción transtorácica del $88 \%$ (78 positiva $+10 \%$ sospechosa). Un $8 \%$ de los pacientes se intervinieron sin poder contar previamente con un diagnóstico histológico de certeza.

Los tipos de cirugía empleados fueron los siguientes: lobectomía (47\%), neumonectomía (34\%), segmentectomía (9\%), bilobectomía (9\%) y resecciones atípicas $(1 \%)$. Se practicaron linfadenectomías en el $76 \%$ de los pacientes $(48 \%$ hiliar y $29 \%$ mediastínica). Un $41 \%$ recibió tratamiento adyuvante con Radioterapia según las indicaciones del protocolo asistencial del Centro. Se empleó, fundamentalmente, en los casos con afectación de los márgenes de resección y/o afectación ganglionar mediastínica.

\section{HALLAZGOS ANATOMO-PATOLÓGICOS}

Los tipos histológicos más comunes fueron el adenocarcinoma (46\%) y el carcinoma epidermoide (42\%). Un $11 \%$ eran carcinomas indiferenciados de células grandes y otros tipos sólo constituyen el $1 \%$ restante. Según el grado de diferenciación histológica, un $28 \%$ eran indiferenciados, un $30 \%$ moderadamente diferenciados y un $42 \%$ bien diferenciados.

La tabla II muestra la estadificación posquirúrgica y su relación con la prequirúrgica. Tres pacientes se intervinieron a pesar de estar en estadio IV (M1). Se trataba de metástasis cerebrales únicas y, siguiendo las indicaciones de nuestro protocolo asistencial, fueron sometidos a una resección quirúrgica de la metástasis, previa a la cirugía pulmonar. Puede observarse que el grado de correlación pre y posquirúrgica es mayor para los estadios I (el $81 \%$ continúan siendo estadios I tras la cirugía) que para los estadio II y IIIA (el 42 y $50 \%$

\begin{tabular}{lccccc}
\multicolumn{7}{c}{ TABLA II } & & \\
\multicolumn{5}{c}{ ESTADIFICACIÓN POSTQUIRÚRGICA } \\
\hline Est. Clínica & $I$ & $\|$ & $I I A$ & $I I B$ & IV \\
\hline$I$ & $35 \%$ & $4 \%$ & $3 \%$ & $1 \%$ & \\
II & $7 \%$ & $11 \%$ & $6 \%$ & $2 \%$ & $1 \%$ \\
IIIA & $4 \%$ & $7 \%$ & $14 \%$ & $3 \%$ & \\
IIIB & & & & & \\
IV & & & & & $2 \%$ \\
\hline
\end{tabular}

mantienen el estadio, respectivamente).

En la tabla III quedan reflejadas ver algunas características histopatológicas relacionadas con la afectación pleural, margen de resección y afectación ganglionar. Es de destacar cómo la probabilidad de afectación neoplásica aumenta drásticamente con el tamaño de los ganglios, desde un $10 \%$ en los menores a $1 \mathrm{~cm}$ hasta el $72 \%$ en los mayores de $2 \mathrm{~cm}$.

\section{RECIDIVA LOCAL}

En la población estudiada se registraron recidivas en el $22 \%$ de los pacientes, en el primero de ellos a los 2 meses y en el último a los 45. La mitad de los que recidivaron lo hicieron antes de los 20 meses. La supervivencia media libre de enfermedad fue de 71 meses (IC95\% 64,4-78,4), no habiéndose

\begin{tabular}{lc}
\multicolumn{2}{c}{ TABLA III } \\
\multicolumn{2}{c}{ HALLAZGOS HISTOPATOLÓGICOS } \\
\hline Características & Frecuencia \\
\hline Afectación pleural: & \\
Ausente & $36 \%$ \\
Subpleural & $17 \%$ \\
Visceral & $36 \%$ \\
Parietal & $6 \%$ \\
Pared costal & $5 \%$ \\
Afectación margen quirúrgico: & \\
Bronquial & $13 \%$ \\
Peritumoral & $10 \%$ \\
Afectación ganglionar (topográfica): & \\
Hiliar & $27 \%$ \\
Intrapulmonar & $11 \%$ \\
Paratraqueal alta & $58 \%$ \\
Paratraqueal baja & $13 \%$ \\
Ventana aorto-pulmonar & $45 \%$ \\
Subcarinal & $16 \%$ \\
Ligamento pulmonar & $13 \%$ \\
Afectación ganglionar por tamaño: & \\
$0-1$ cm & $10 \%$ \\
1-2 cm & \\
$>2$ cm & $55 \%$ \\
\hline
\end{tabular}

alcanzado la mediana en el momento de la conclusión del estudio. La SLE estimada a 3 años fue del $70 \%$ (Error Estándar: 0,04).

\section{ANÁLISIS ESTADÍSTICO GENERAL}

En primer lugar se realizó un análisis univariante comparando la supervivencia libre de enfermedad entre grupos de pacientes definidos por factores, tanto clínicos (prequirúrgicos) como histopatológicos (posquirúrgicos). De los factores clínicos, sólo el contacto radiológico del tumor o sus espiculaciones con la pleura, visible en la TAC, mostró un valor pronóstico estadísticamente significativo $(p=0,005)$. No se encontraron diferencias significativas en la SLE de los enfermos según la edad, segundas neoplasias, diagnóstico casual (asintomáticos), realización de punción transtorácica diagnóstica, características radiológicas de cavitación y presencia de espiculaciones. Tampoco influyó el tipo de intervención quirúrgica empleado.

En el análisis de las características anatomo-patológicas, encontramos que el tipo histológico, tamaño tumoral y afectación del margen peribronquial, no influyeron en la SLE. Por el contrario, sí mostraron valor pronóstico sobre el tiempo hasta la recidiva los siguientes factores: Grado de diferenciación, afectación del margen peritumoral, infiltración pleural, estadificación $\mathrm{T}$ y $\mathrm{N}$.

Con estas últimas variables se realizó un análisis de regresión de Cox. La diferenciación celular, así como la afectación pleural y ganglionar, mantienen su valor pronóstico en el estudio multivariante; no así la estadificación $\mathrm{T}$, ni la afectación del margen quirúrgico peritumoral, como se muestra en la tabla IV. Puede verse que los tumores indiferenciados tienen 4,2 veces más riesgo de recidiva local que los bien diferencia- 


\begin{tabular}{lccc}
\multicolumn{5}{c}{ TABLA IV } \\
\multicolumn{4}{c}{ REGRESIÓN DE COX } \\
\hline Variable & Riesgo & IC95\% & p-valor \\
\hline Grado diferenciación & & & \\
G1 & 1 & - & 0,03 -global \\
G2 & 1,8 & $0,4-7$ & 0,3 \\
C3 & 4,2 & $1,1-5$ & 0,029 \\
Afectación pleural & 1 & & 0,0014 -global \\
ausente & 1,3 & $1,3-13$ & 0,012 \\
pl. visceral & 13 & $3,2-53$ & 0,0003 \\
pl. parietal / costal & & & \\
Estadificación N & 1 & - & 0,02 -global \\
N0 & 2,9 & $1-8$ & 0,048 \\
N1 & 3,7 & $1-10$ & 0,009 \\
N2 & & & \\
\hline
\end{tabular}

dos, con medianas de SLE de 50 (IC95\% 41-60) y 82 meses (IC95\% 72-9) respectivamente. La infiltración de la pleura parietal o pared costal, multiplica por 13 el riesgo de recidiva y la afectación de la pleura visceral lo hace por 4,3. La mediana de la SLE es de 58 meses (IC95\% 46-69) cuando hay algún tipo de afectación pleural, frente a 84 meses (IC95\% 76-92) cuando está libre de infiltración. El riesgo de recaída local es 3,7 veces superior para los pacientes con afectación ganglionar $\mathrm{N} 2$ y 2,9 veces para los pacientes con $\mathrm{N} 1$, con relación a los N0. Las medianas de SLE fueron de 33 meses (IC95\% 2343) para los N2, 44 meses (IC95\% 33-56) para los N1 y 80 meses (IC95\% 72-88) para los N0.

En las figuras 1, 2 y 3 pueden verse representaciones gráficas de las diferencias entre las curvas de supervivencia libre de enfermedad según estos factores pronósticos.

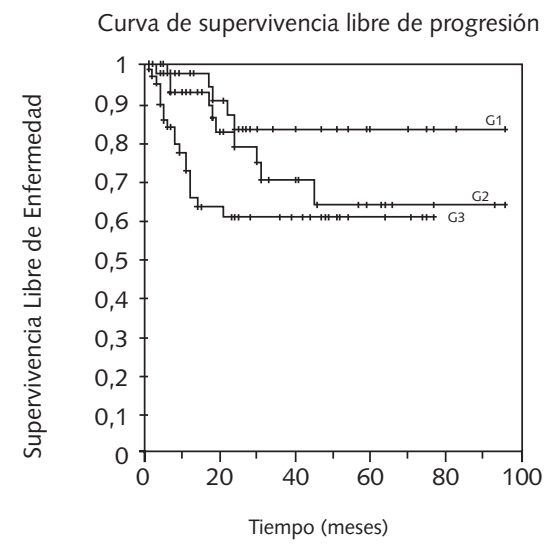

Fig. 1. Supervivencia libre de enfermedad actuarial por grupos, según el grado de diferenciación histológica. Prueba de los rangos logarítmicos: $p=0,03$.

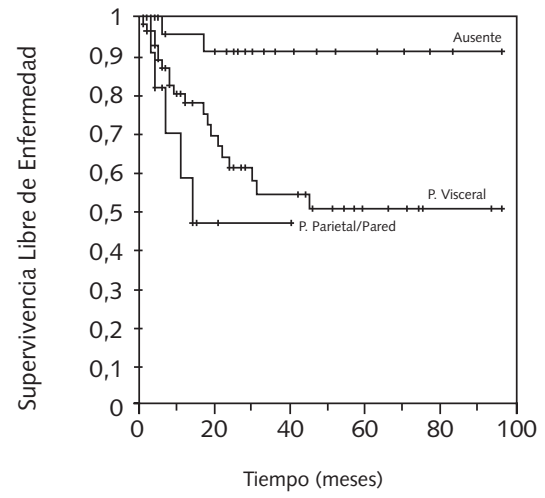

Fig. 2. Supervivencia libre de enfermedad actuarial por grupos, según el grado de afectación pleural. Prueba de los rangos logarítmicos: $p=0,006$.

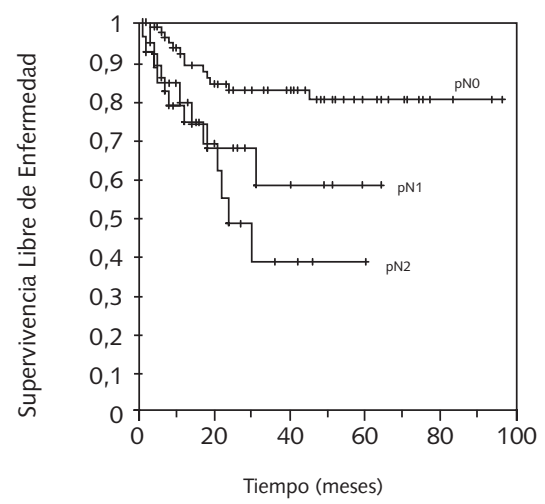

Fig. 3. Supervivencia libre de enfermedad actuarial por grupos, según la afectación ganglionar (N). Prueba de los rangos logarítmicos: $p=0,002$.

\section{DISCUSIÓN}

Las características generales de los pacientes incluidos en este estudio (edad, tipo histológico, estadificación) no difieren mucho de las de otros estudios1 de similares características. Ahora bien, comparado con un estudio previo realizado en el mismo Centro (14), sobre el mismo tipo de pacientes (carcinoma no microcítico de pulmón) y en el mismo periodo de tiempo, pero tratados con Radioterapia radical, encontramos algunas diferencias. El grupo tratado con Radioterapia eran de edades algo mayores (64 vs. 61) y con una enfermedad más avanzada. También hay diferencias en el tipo histológico, con predominio del tipo epidermoide (54\%) en el grupo de pacientes tratado con Radioterapia frente al predominio de adenocarcinomas (46\%) en el presente estudio, aunque hay que hacer notar que el método diagnóstico empleado es, obviamente, distinto.

El 16\% de los enfermos tenían antecedentes de otra neoplasia y un tercio no presentaban signos ni síntomas relacionados con la enfermedad. Estos datos invitan a meditar sobre el 
empleo de pruebas de cribado radiológico de tórax, en pacientes con otras neoplasias relacionadas con el consumo de tabaco (tumores del tracto aero-digestivo superior y urinario), si bien en el momento actual no existe una clara evidencia de su utilidad (15).

Los factores pronósticos más importantes de recidiva local fueron la infiltración pleural, la afectación ganglionar y el grado de diferenciación histológica. El grado de afectación pleural es un factor pronóstico bien conocido (16-18) y en esta serie ha sido el más determinante para predecir la recaída local. También, como otros autores (19-21), se ha encontrado una alta correlación de estos factores entre sí que, por otro lado, mantienen su valor pronóstico en el estudio multivariante. Por tanto, puede afirmarse que se trata de factores independientes, si bien con frecuencia se presentan juntos, ya que estarían describiendo grupos de pacientes con un perfil pronóstico similar. Otros autores (22), sin embargo, establecen una relación de causalidad, de modo que la pleura visceral constituiría una "barrera" anatómica para la diseminación linfática e incluso proponen una modificación en la clasificación TNM con objeto de incluir un estadio IC para tumores N0 con infiltración de la pleura visceral. En el estudio de Suzuki y cols. (21), sobre 389 pacientes en estadio clínico IA intervenidos, se encuentra una estrecha relación entre grado histológico de diferenciación, afectación pleural y la afectación ganglionar patológica que mostraron el $23 \%$ de los enfermos, con consecuencias en el pronóstico. Otro grupo japonés (Okada y cols.) (23) ha publicado recientemente su experiencia con lavado pleural intraoperatorio en 1000 pacientes consecutivos sin evidencia de derrame pleural y demuestran una mayor tasa de recidivas y peor supervivencia en el $4,5 \%$ de los pacientes que mostraron citología positiva del lavado.

En esta serie, la infiltración de la pleura ha mostrado un valor pronóstico muy superior al resto de las variables analizadas, incluida la estadificación TNM. De hecho, la única variable clínica pre-quirúrgica con influencia en la recidiva ha sido el contacto radiológico del tumor con la pleura. Probablemente, esta fuerza pronóstica oscurezca la influencia de otros factores como el tipo histológico y tamaño tumoral, que, sin embargo, han demostrado un valor pronóstico en otros estu-

\section{Bibliografía}

1. Grupo de Estudio del Carcinoma Broncopulmonar de la SOCALPAR Incidencia del carcinoma broncopulmonar en Castilla-León durante el año 1997. Estudio multicéntrico de la Sociedad Castellano-Leonesa de Patología Respiratoria (SOCALPAR). Arch Bronconeumol 2000; 36: 313-8.

2. Egermann U, Jaeggi K, Habicht JM, Perruchoud AP, Dalquen P, Soler M. Regular follow-up after curative resection of nonsmall cell lung cancer: A real benefit for patients? Eur Respir J 2002; 19: 464-8.

3. Ravdin PM, Davis G. Prognosis of patients with resected non-small cell lung cancer: Impact of clinical and pathologic variables. Lung Cancer 2006; 52: 207-12.

4. Sugimura H, Nichols FC, Yang P, Allen MS, Cassivi SD, Deschamps C et al. Survival after recurrent nonsmall-cell lung cancer after complete pulmonary resection. Ann Thorac Surg 2007 ;83 : 409-18.

5. Palacios Calvo J. Factores pronósticos diferentes del TNM. Rev Patol Respir 2000; 3: 32-4.

6. Firat S, Byhardt RW, Gore E. Comorbidity and Karnofsky Performance score are independent prognostic factors in stage-III non-small-cell lung cancer: An institutional analysis of patients treated of four RTOG studies. Int J Radiat Oncol Biol Phys 2002; 54: 357-64.

7. Mountain CF: Revisions in the International System for Staging Lung dios. Así, Wertzel y cols. (24) encuentran una tasa mayor de recidiva en los adenocarcinomas respecto a los epidermoides y lo justifican porque los primeros, con mayor tendencia a localizarse periféricamente, infiltrarían la pleura con mayor frecuencia. Aunque podría pensarse algo parecido respecto al tamaño tumoral, de forma que tumores más grandes tendrían más posibilidades de afectar la pleura, hay estudios, como el de Harpole y cols. (25), que demuestran una mayor incidencia de recidiva en tumores mayores a $3 \mathrm{~cm}$, independientemente del estadio. Otro estudio nacional reciente (25) de pacientes con tumores en estadio I (menores a $3 \mathrm{~cm}$ ) muestra distinta supervivencia entre tumores menores a $2 \mathrm{~cm}$ y aquellos comprendidos entre 2 y $3 \mathrm{~cm}$, independientemente de otros posibles factores.

En cuanto a la afectación de los márgenes quirúrgicos, este es un factor controvertido (27-30). En esta serie la afectación del margen peribronquial no influyó en la recidiva local, si bien hay que señalar que ésta fue una indicación de Radioterapia postoperatoria. Por otro lado, la afectación del margen peritumoral perdió su valor pronóstico en el estudio multivariante, lo que nos lleva a pensar que puede tratarse de un factor meramente "acompañante" de otros. La afectación ganglionar, hiliar o mediastínica, influyó claramente en la recidiva, hecho sobradamente conocido y, de hecho, incluido en la estadificación TNM (N1-N2). El grado de diferenciación histológica también influyó en la recaída local, en sintonía con lo encontrado por otros autores (31-33). Los tumores más indiferenciados tienen mayor probabilidad de recidivar.

Como conclusión, en los pacientes diagnosticados de carcinoma no microcítico de pulmón y susceptibles de tratamiento quirúrgico, debemos considerar otros factores pronósticos aparte de la estadificación TNM, como son el grado de diferenciación histológica y la afectación pleural. En este sentido, podría resultar muy interesante promover ensayos clínicos controlados para evaluar la utilidad de otras terapias alternativas o complementarias a la cirugía (radioterapia, quimioterapia) en pacientes con especial mal pronóstico, esto es, con tumores indiferenciados y con signos de afectación pleural y ganglionar.
Cancer. Chest 1997; 111: 1710-7.

8. Lung. In: American Joint Committee on Cancer: AJCC Cancer Staging Manual. Philadelphia: Lippincott-Raven Publishers, $5^{\text {th }}$ ed. 1997: 12737.

9. Grupo de Trabajo de la SEPAR. Normativa actualizada (1998) sobre diagnóstico y estadificación del carcinoma broncogénico. Arch Bronconeumol 1998; 34: 437-52.

10. Miller AB, Hoogstraten B, Staquet M, Winkler A. Reporting results of cancer treatment. Cancer 1981; 47: 207-14.

11. Kaplan EL, Meier P. Nonparametric estimation from incomplete observations. J Amer Statist Assoc 1958; 55: 457-81.

12. Mantel N. Evaluation of survival data and two new rank order statistics arising in its consideration. Cancer Chemother Rep 1966; 50: 163-70.

13. Cox DR. Regression models and life tables. J Roy Statist Soc 1972; 34, series B: 187-220.

14. Jaén J, Alonso A, León A, Rueda A. Carcinoma no microcítico de pulmón. Supervivencia y factores pronósticos del tratamiento radioterápico. Arch Bronconeumol 2003; 39: 81-6.

15. Manser R, Irving L, Stone C, Byrnes G, Abramson M, Campbell D Screening for lung cancer. Cochrane Database Syst Rev 2004; 1: CD001991. 
16. Fujimoto T, Cassivi SD, Yang P, Barnes SA, Nichols FC Deschamps C et al. Completely resected N1 non-small cell lung cancer: Factors affecting recurrence and long-term survival. Jour Thorac Cardiovasc Surg 2006; 3: 499-506.

17. Niizeki H, Morikawa T, Okushiba S, Kondo S, Katoh H. Survival and prognostic factors in resected cN2-pN0 non-small cell lung cancer. Ann Thorac Cardiovasc Surg 2004; 10: 9-13.

18. Yoshino I, Yamaguchi M, Tagawa T, Fukuyama S, Kameyama T, Osoegawa A, et al. Operative results of clinical stage I non-small cell lung cancer. Lung Cancer 2003; 42: 221-5.

19. Chapelier A, Fadel E, Macchiarini P, Lenot B, Le Roy Ladurie F, Cerrina $\mathbf{J}$, et al. Factors affecting long-term survival after en-bloc resection of lung cancer invading the chest wall. Eur J Cardiothorac Surg 2000; 18: 513-8.

20. Kang JH, Kim KD, Chung KY. Prognostic value of visceral pleura invasion in non-small cell lung cancer. Eur J Cardiothorac Surg 2003 23: 865-9.

21. Suzuki K, Nagai K, Yoshida J, Nishimura M, Nishiwaki Y. Predictors of lymph node and intrapulmonary metastasis in clinical stage IA nonsmall cell lung carcinoma. Ann Thorac Surg 2001; 72: 352-6.

22. Manac'h D, Riquet M, Medioni J, Le Pimpec-Barthes F, Dujon A, et Danel C. Visceral Pleura Invasion by Non-Small Cell Lung Cancer: An underrated bad prognostic Factor. Ann Thorac Surg 2001; 71: 1088-93.

23. Okada M, Sakamoto T, Nishio W, Uchino K, Tsuboshima K, Tsubota N. Pleural lavage cytology in non-small cell lung cancer: Lessons from 1000 consecutive resections. J Thorac Cardiovasc Surg 2003; 126: 1911-5.

24. Wertzel H, Siebert H, Lange W, Swoboda L, Graf E, Hasse J. Results after surgery in stage I bronchogenic carcinoma. Thorac Cardiovasc Surg 1998; 46: 365-69.

25. Harpole D, Herdon JE, Young WG, Wlfe WG, Sabiton DC. Stage I nonsmall cell lung cancer. A multivariate analysis of treatment methods and patterns of recurrence. Cancer 1995; 76: 787-896.

26. Padilla J, Calvo V, Peñalver JC, Jordá C, Escrivá J, García A, et al. Carcinoma broncogénico no anaplásico de células pequeñas en estadio I y de diámetro máximo de $3 \mathrm{~cm}$. Factores pronósticos. Arch Bronconeumol 2004; 40: 110-3

27. Carbone E, Spinelli E, Droz Blanc C, Cascino QR, Motta G. Considerations about the complete resection in NSCLC surgery. Review. Ann Ital Chir 2002; 73: 365-74.

28. Hofmann HS, Taege C, Lautenschlager C, Neef H, Silber RE. Microscopic (R1) and macroscopic (R2) residual disease in patients with resected non-small cell lung cancer. Eur J Cardiothorac Surg 2002; 21: 606-10.

29. Lequaglie C, Conti B, Brega Massone PP, Giudice G. Unsuspected residual disease at the resection margin after surgery for lung cancer: Fate of patients after long-term follow-up. Eur J Cardiothorac Surg 2003; 23: 229-32.

30. Wick MR, Mills SE. Evaluation of Surgical Margins in Anatomic Pathology: Technical, Conceptual and Clinical Considerations. Seminars in Diagnostic Pathol 2002; 19: 207-8.

31. Mehdi SA, Tatum AH, Newman NB, Gamble GP, Etzell JE, Weidner $\mathrm{N}$, et al. Prognostic markers in resected stage I and II non small-cell lung cancer: An analysis of 260 patients with 5 year follow-up. Clin Lung Cancer 1999; 1: 59-67.

32. Moldvay J, Scheid P, Wild P, Nabil K, Siat J, Borrelly J, et al. Predictive survival markers in patients with surgically resected non-small cell lung carcinoma. Clin Cancer Res 2000; 6: 1125-34.

33. Gawrychowski J, Gabriel A, Lackowska B. Heterogeneity of stage IIIA non-small cell lung cancers (NSCLC) and evaluation of late results of surgical treatment. Eur J Surg Oncol 2003; 29: 178-84. 